\title{
Highlights from the heavy-ion program in STAR
}

\author{
Petr Chaloupka ${ }^{1, a}$ for the STAR collaboration \\ ${ }^{1}$ Czech Technical University in Prague, Faculty of Nuclear Sciences and Physical Engineering
}

\begin{abstract}
The experiments at RHIC have produced convincing evidence that strongly interacting partonic matter is created in the collisions of heavy ions. The unique flexibility of RHIC to collide different nuclear species over a wide range of collision energies together with STAR's wide acceptance and particle identification are ideally suited for systematic exploration of the properties of this QCD matter.

STAR collaboration has successfully completed the Beam Energy Scan, program focused on searching for the onset of the QGP signatures and studying the nature of the phase transition, indicating that the region of interests for critical point and the first-order phase transition is within the reach of RHIC experiments. Moreover, with its two newly installed detector upgrades, STAR has launched a comprehensive heavy-flavor program which allows high precision measurements of the properties of the partonic matter.
\end{abstract}

\section{Introduction}

Collisions of heavy ions at ultra-relativistic energies have been used to create a new state of matter called the "Quark Gluon Plasma (QGP)", in which deconfined quarks and gluons are the relevant degrees of freedom $[1,2]$. The detailed studies of this strongly interacting matter, as it is currently performed by the STAR experiment at RHIC, provide important tests of Quantum Chromodynamics (QCD) and its emergent properties.

The RHIC's unique capability to collide different nuclear species over a broad range of collision energies allows to systematically explore the QCD phase diagram. At the highest RHIC collision energies the system at mid-rapidity attains the highest temperature and vanishingly small net baryon density. In these collisions the high statistics data, which were collected thanks to luminosity upgrades of RHIC, are used to study the quantitative properties of the QGP such as viscosity, transport parameters, heavy quark diffusion coefficients, and others. At lower collision energies, where baryon density is finite, STAR experiment seeks to answer questions about the actual phase transition from the hadronic matter into the QGP, most importantly: where is the onset of the QGP formation? What is the nature of the phase transition and is there a critical point in the QCD phase diagram?

In order to reach these goals STAR collaboration has successfully finished two major detector upgrades. In 2014 the Muon Telescope Detector (MTD) [3] was fully installed in STAR. Its purpose is detection and identification of muons with $p_{T}>1.2 \mathrm{GeV} / \mathrm{c}$ and $|y|<0.5$. In the same year was also installed a new vertex detector called Heavy Flavor Tracker (HFT) [4]. This high-precision inner

ae-mail: petr.chaloupka@fjfi.cvut.cz 
tracker can determine low momentum open heavy quark decay vertices in the environment of highmultiplicity heavy-ion collisions. These upgrades have significantly enhanced STAR's capabilities for heavy flavor measurements including open heavy quark hadrons and quarkonia. Moreover from 2010 to 2014 RHIC has conducted a Beam Energy Scan (BES) program aimed at exploring the nuclear matter phase diagram. In this review we highlight and discuss some of the results from the BES program as well as results obtained using the two new STAR upgrades.

\section{Results from Beam Energy Scan}

The Beam Energy Scan (BES) program has been carried out at RHIC in order to study the structure of the QCD phase diagram. The program has covered beam energies of $\sqrt{s_{N N}}=7.7,11.5,14.5,19.6$, $27,39 \mathrm{GeV}$ between the years 2010 to 2014 , together with $62.4,130,200 \mathrm{GeV}$ from previous years. This collision energy range means that the STAR experiment can effectively map the QCD phase diagram in the region of baryon chemical potential of $20<\mu_{B}<420 \mathrm{MeV}$. This detailed exploration is expected to reveal several key features of the QCD phase diagram[5, 6].

It is well established that at the highest collision energy the strongly coupled QGP has been created $[1,2]$. The created medium shows a strong opacity to colored probes and very small ratio of shear viscosity over entropy density. Lowering the collision energy allows to study the emergent properties of the partonic matter and search for the onset of its formation.
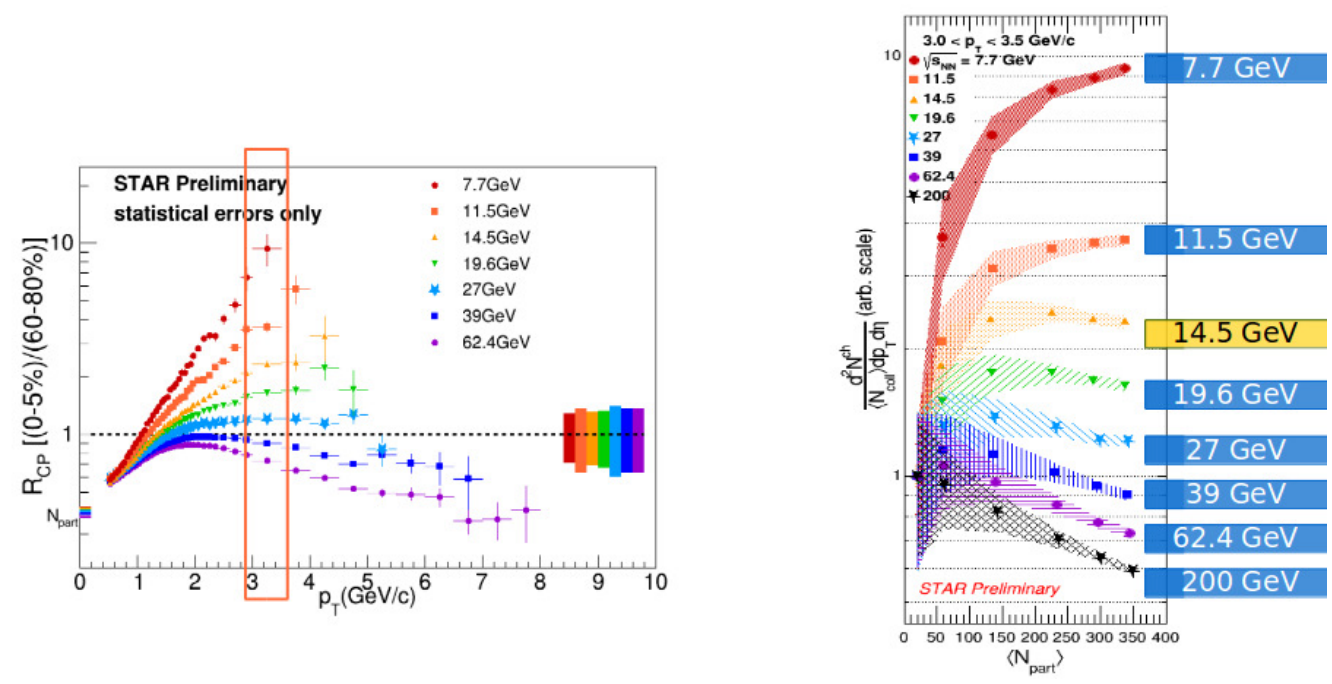

Figure 1. (Left) Charged hadron $R_{C P}$ in $\mathrm{Au}+\mathrm{Au}$ collisions at different energies. (Right) Integrated yields $(3<$ $p_{T}<3.5 \mathrm{GeV}$ ) per number of binary collisions as a function of number of participants at different collision energies. The yields are normalized so that the yield in most peripheral bin is unity.

The suppression of high $p_{T}$ hadrons is one of the most pronounced signatures of energy loss of partons traversing the QGP. Figure 1 shows on the left a nuclear modification factor $R_{C P}$ (the binary-collision-scaled $p_{T}$ yields) of charged hadron at different BES energies [7]. As can be seen the $R_{C P}$ transitions from a strong suppression at high energies to an enhancement at lower beam 
energies. However, the measured value of $R_{C P}$ is also affected by competing enhancement from Cronin effect which gets stronger at lower collision energies. This means that QGP can be formed even at collision energies where $R_{C P}$ is not below one. More differential study can help to disentangle these contributions. In right part of Figure 1 are shown STAR preliminary results on $p_{T}$ integrated yields $\left(3<p_{T}<3.5 \mathrm{GeV}\right)$ per number of binary collisions [7] as a function of number of participants. For every collision energy the yields are scaled by the corresponding most peripheral bin. As expected the suppression effects quickly dominate at high collision energies. On the other hand at lowest energies the enhancement is clearly present in the most central collisions. The competing effects approximately cancel each other at the at $\sqrt{s_{N N}}=14.5 \mathrm{GeV}$.

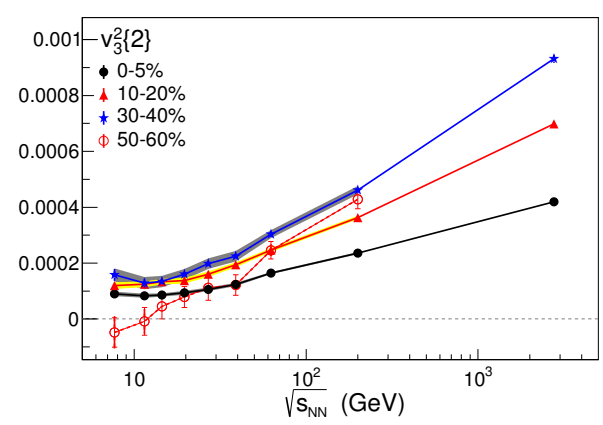

Figure 2. Collision energy dependence of $v_{3}^{2}\{2\}$ in $\mathrm{Au}+\mathrm{Au}$ collisions from STAR and in $\mathrm{Pb}+\mathrm{Pb}$ collisions from ALICE [8].

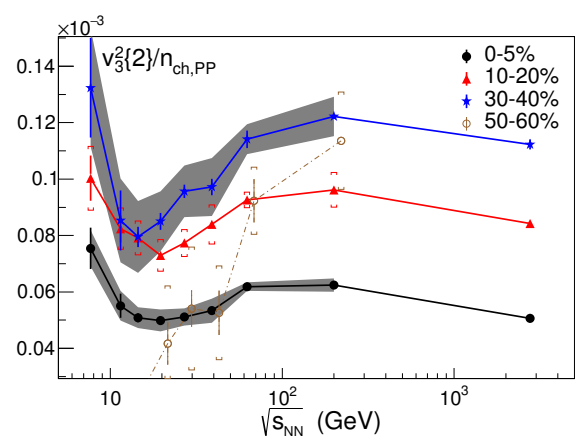

Figure 3. Collision energy dependence of $v_{3}^{2}\{2\}$ normalized by the multiplicity per participant pair $\left(n_{c h, P P}=d N_{c h} / d y /\left(N_{\text {part }} / 2\right)\right.$.

Another signature related to the existence of the QGP phase is the observation of triangular flow $v_{3}$ [9] since its non-zero value requires the presence of a low viscosity phase early in the collision. STAR has recently published results on measurements of $v_{3}^{2}\{2\}$ from the BES [10]. In Figure 2 are shown STAR measurements of charged hadron $v_{3}^{2}\{2\}$ in Au+Au together with ALICE measurement in $\mathrm{Pb}+\mathrm{Pb}$ collisions at $\sqrt{s_{N N}}=2.76 \mathrm{TeV}$ [8]. In central and mid-central collisions we observe nonzero $v_{3}$ at collision energies which may suggest that the QGP phase exists even at the low collision energies of the BES. On the other hand, in peripheral collisions the observed $v_{3}$ is consistent with zero for energies below $14.5 \mathrm{GeV}$. It is expected that the ability of the system to convert initial state geometry fluctuations into the final state $v_{3}$ scales with multiplicity of the produced particles. Deviations from that expectation could indicate interesting physics like a softening of the equation-of-state [11]. Figure 3 shows energy dependence of $v_{3}^{2}\{2\}$ divided by the multiplicity per participant pair $\left(n_{c h, P P}=d N_{c h} / d y /\left(N_{p a r t} / 2\right)\right.$. The $n_{c h, P P}$ is proportional to the system energy density. As can be seen the data show a local minimum in the region of $\sqrt{s_{N N}}=15-20 \mathrm{GeV}$ which could indicate an interesting trend in the pressure developed inside the system.

One of the main motivations for the BES program at RHIC is to search for signatures of the firstorder phase transition and the possible QCD critical point. It has been argued that observation of minimum in the slope of directed flow $\left(d v_{1} / d y\right)$ of protons and its double sign-change for net protons in the STAR data [12] can be linked to the softening of the equation of state [13]. So far models had hard time reproducing this structure in $d v_{1} / d y$ which is expected to result from an interplay between hydro, baryon transport dynamics and baryon/anti-baryon annihilation. STAR has recently measured 


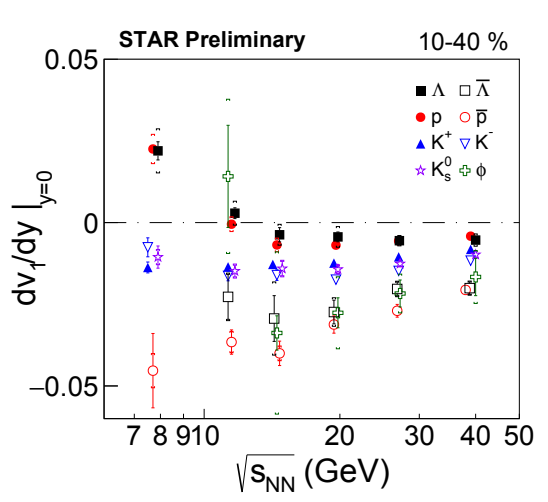

Figure 4. Beam energy dependence of $d v_{1} / d y$ for $p, \bar{p}, K^{ \pm}, K_{s}^{0}, \phi, \Lambda$ and $\bar{\Lambda}$ in $10-40 \% \mathrm{Au}+\mathrm{Au}$ collisions.

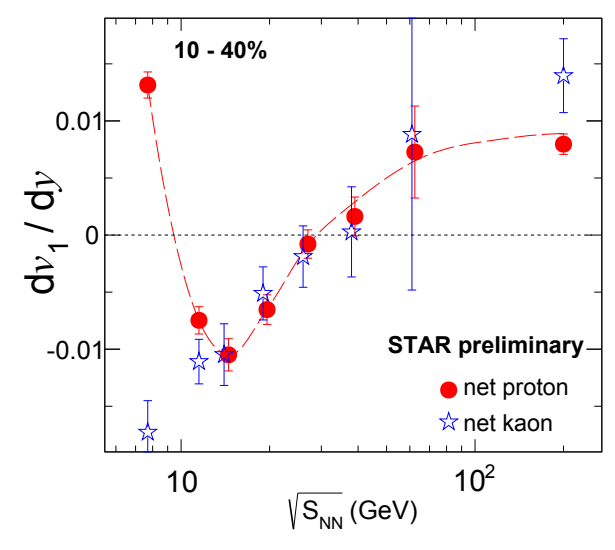

Figure 5. Beam Energy dependence of net-particle $d v_{1} / d y$ in $10-40 \% \mathrm{Au}+\mathrm{Au}$ collisions.

directed flow of $\Lambda, \bar{\Lambda}, K^{ \pm}, K_{S}^{0}$ and $\phi$ in Au+Au collisions at the BES energies [14]. Since these particles contain different constituent quarks the measurements will help to understand the QCD phase transition and it will allow to disentangle the role of produced and transported quarks in heavy-ion collisions.

Figure 4 shows the $d v_{1} / d y$ for different particle species as measured by the STAR experiment. As can be seen, the $d v_{1} / d y$ of $\Lambda$ is consistent with that of the proton and similarly shows a change in sign near $\sqrt{s_{N N}}=11.5 \mathrm{GeV}$. For the higher collision energies above $14.5 \mathrm{GeV}$, where antibaryon production is significant, the $\bar{\Lambda}$ and $\phi$ follow the trend of antiprotons above $14.5 \mathrm{GeV}$. However, below 14.5 GeV the $d v_{1} / d y$ of $\phi$ is, within large uncertainties, consistent with zero. Figure 5 shows collision energy dependence of net-proton and net-kaon $d v_{1} / d y$. Such a study is expected to be sensitive to the difference between the contributions from produced quarks and quarks that are transported from the initial state. As can be seen the net-p and net-K $d v_{1} / d y$ follow a similar trend for $\sqrt{s_{N N}}=14.5-200$ $\mathrm{GeV}$. At lower collision energies they start to strongly deviate from each other.

The type of the transition between the hadronic and partonic phase depends on where we are in the QCD phase diagram with regard to the temperature and the baryonic chemical potential of the system. While smooth cross-over is expected at the vanishing baryonic chemical potential, at higher net-baryon region model calculations predict the existence of a first-order phase transition. This implies that there should be a critical point between these two regions [15].

One of the main goals of BES at the RHIC is to study the QCD phase diagram over a wide range of chemical potential $\left(\mu_{B}\right)$ and search for the QCD critical point. The critical point is characteristic by large correlation length of the dynamical system which manifests in large event-by-event fluctuations of conserved quantities, such as net-baryon number (B), net-charge $(\mathrm{Q})$, and net-strangeness $(\mathrm{S})[16-$ 18]. The experimentally accessible order moments of these quantities, variance $\left(\sigma^{2}\right)$, skewness $(\mathrm{S})$, and kurtosis $(\kappa)$, are directly connected to the theoretically calculated thermodynamic susceptibilities. However, since it is very hard to measure the net-baryon $\left(\Delta N_{B}\right)$ and the net-strangeness $\left(\Delta N_{S}\right)$ distributions it is common to use net-proton $\left(\Delta N_{P}\right)$ and net-kaon $\left(\Delta N_{K}\right)$ as proxies. STAR has performed analysis of cumulants and cumulant ratios of the net-proton, net-kaon and net-charge multiplicity distributions in $\mathrm{Au}+\mathrm{Au}$ collisions from BES [14]. Figure 6 shows the energy dependence of the vol- 

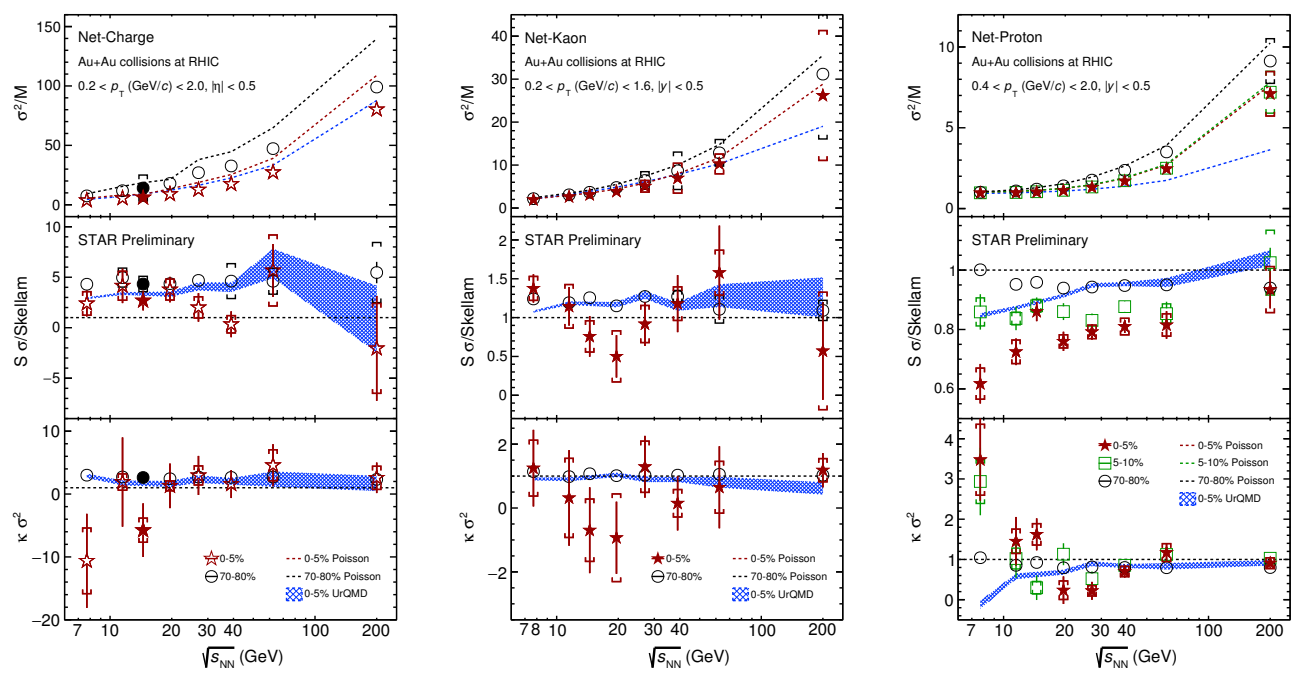

Figure 6. Energy dependence of cumulant ratios $\left(\sigma^{2} / M, S \sigma / S k e l l a m, \kappa \sigma^{2}\right)$ of net-charge, net-kaon, and netproton multiplicity distributions for top 0-5\% central (red stars), 5-10\% central (green squares), and 70-80\% peripheral (black circles) collisions. The Poisson expectations are denoted as dotted lines and UrQMD calculations are shown as blue bands.

ume independent cumulant ratios $\left(\sigma^{2} / M, S \sigma / S\right.$ kellam, $\left.\kappa \sigma^{2}\right)$ for net-proton, net-kaon, and net-charge multiplicity distributions in Au+Au collisions from BES as measured by the STAR experiment. The experimental data are compared to predictions from UrQMD calculations (blue bands) which do not include critical physics processes. The net-charge and net-kaon cumulants are observed to be within uncertainties consistent with Poisson expectations (dashed lines in the figure). Both the data and the UrQMD simulations show no energy dependence for $S \sigma^{2} /$ Skellam and $\kappa \sigma^{2}$ in net-charge and net-kaon measurement. However, the values of net-proton $S \sigma^{2} /$ Skellam and $\kappa \sigma^{2}$ in central collisions clearly exhibit a deviation from the Poisson expectation of unity as well as from the UrQMD calculations. More precise systematic studies will be possible in future with the data from the planned second phase of the Beam Energy Scan (BES-II).

\section{Measurements with Heavy Flavor Tracker}

Heavy quarks which are predominantly produced in the early stages of the collision experience the full evolution of the system while being mostly unaffected by the QCD medium due to their large masses. Study of heavy quark propagation through the QGP gives an information about transport properties of the medium.

Heavy Flavor Tracker (HFT) micro-vertexing detector has been built and installed into STAR successfully before the RHIC year 2014 running. The HFT consists of four layers of silicon detectors. While outer layers are silicon strip detectors the two innermost layers are made from state-of-theart ultra-thin CMOS Monolithic Active Pixel Sensors (MAPS). The HFT is a first application of the CMOS MAPS detector in a collider experiment. The proximity to the collision location and the low material budget allow HFT to achieve a track pointing resolution of less than $50 \mu \mathrm{m}$ with $p_{T}=750 \mathrm{MeV} / \mathrm{c}$. 

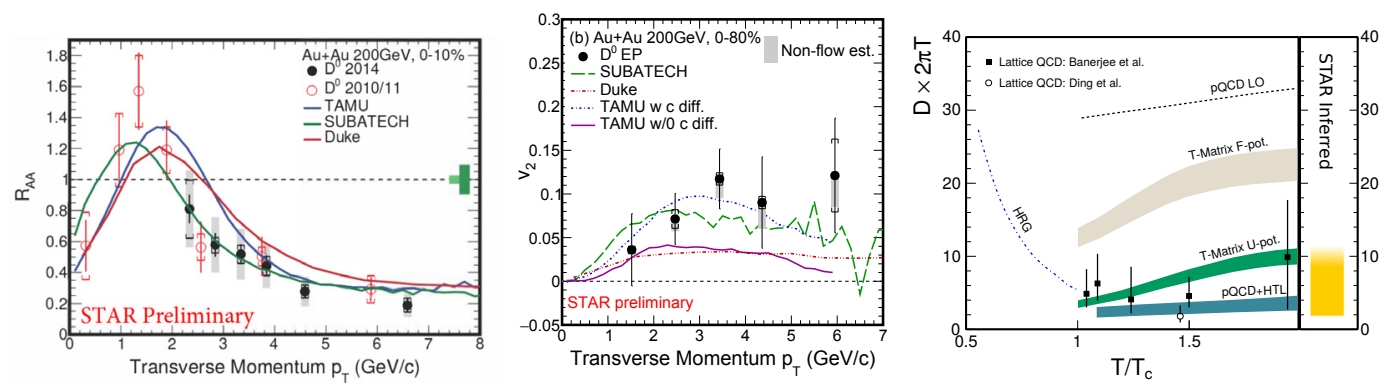

Figure 7. STAR results on nuclear Figure 8. Comparison of $D^{0}$ Figure 9. Diffusion coefficient from modification factor $R_{A A}$ of $D^{0}$ with and $v_{2}$ in $0-80 \%$ most central events model calculations and the inferred without the HFT, compared to pions. with model calculations. range from STAR measurements.

STAR has reported preliminary results on open charm production in $\mathrm{Au}+\mathrm{Au}$ collisions at $\sqrt{s_{N N}}=200 \mathrm{GeV}$ from analysis where charmed mesons were topologically reconstructed using the HFT $[19,20]$. In the Figure 7 are shown results on $D^{0} R_{A A}$ in $0-10 \%$ most central Au+Au collisions at $\sqrt{s_{N N}}=200 \mathrm{GeV}$. Open red circles denote already published STAR results from 1.1 billion events recorded in 2010 and 2011 without the HFT [21]. Solid black circles show the recent measurement with the HFT from approximately 780 million minimum bias events. The use of HFT significantly improves the precision and results clearly confirm the strong suppression of $D^{0}$ production at high $p_{T}$. The enhancement in the low- $p_{T}$ region can be explained by coalescence of charmed quarks with a flowing medium.

STAR has also performed first measurements of elliptic flow of open charm hadrons at RHIC [19, 20, 22]. In Figure 8 is shown $D^{0} v_{2}$ in $0-80 \% \mathrm{Au}+\mathrm{Au}$ collisions extracted with the use of HFT. Significant non-zero $v_{2}$ is observed for $p_{T}>2 \mathrm{GeV} / \mathrm{c}$. However, its magnitude is systematically lower than $v_{2}$ of lighter hadrons, as discussed in detail in [20].

These measurements of heavy-quark energy loss and elliptic flow allow to extract transport coefficients as a further characterization of the medium. Both Figures 7, and 8 show comparison to model calculations which include charm diffusion [23-25]. A detailed description of the models and comparison to data can be found in [20]. Figure 9 shows the comparison of extracted diffusion coefficient from the different model calculations compared to the range of inferred values that are compatible with the presented STAR measurements (yellow band in the figure). The compatible values of the charm diffusion coefficient $2 \pi T D_{S}$ are between 2 and 12 which is also compatible with results from Lattice QCD.

\section{Quarkonia measurements with MTD}

Suppression of heavy quarkonia due to Debye-like screening of the quark-antiquark potential, has been predicted to be an indicator sensitive to the presence of the QGP [26]. The Debye screening length depends on the temperature attained by the QGP medium. Since different quarkonium states have different binding energies (hence sizes), they are expected to melt at different QGP temperatures. The observed quarkonium suppression pattern can then be linked to the thermodynamical properties of the created QGP.

However, there are other effects that can alter the quarkonium production with respect to the baseline of $\mathrm{p}+\mathrm{p}$ collisions. These effects include secondary production of quarkonia in the QGP via 
heavy quark recombination together with cold nuclear matter effects (CNM) such as shadowing/antishadowing of parton distribution functions, initial-state parton energy loss and final state nuclear absorption. Since these mechanisms have different dependencies on variables such as $p_{T}$ or energy density, differential measurements of quarkonium production at different collision energies, collision systems, and centralities can help to disentangle the interplay of these effects and to study properties of the created medium.

It should be noted that the experimentally measured yields of $J / \psi$ and $\Upsilon$ contain contributions from feed-down. Inclusive $J / \psi$ production is a combination of prompt and non-prompt $J / \psi$. The prompt $J / \psi$ are either direct $(\sim 60 \%)$ or from feed-down from higher excites states $\left(\psi(2 S)\right.$ and $\left.\chi_{C}\right)$. The non-prompt $J / \psi$ originate from B-hadron decays. This contribution has been estimated by the STAR experiment to be $10 \sim 25 \%$ in the range of $4<p_{T}<12 \mathrm{GeV} / c$ [27]. It is hence important to study the production of higher charmonium states in both $\mathrm{p}+\mathrm{p}$ and nucleus-nucleus collisions.

The Muon Telescope Detector (MTD) [3] which was fully installed in 2014 is designed to identify muons and also to provide triggering on di-muons from heavy flavor decays. This allows STAR to perform high-precision quarkonia measurement at mid-rapidity in the di-muon channel which is of an advantage since the di-muon decay channel is less affected by bremsstrahlung.

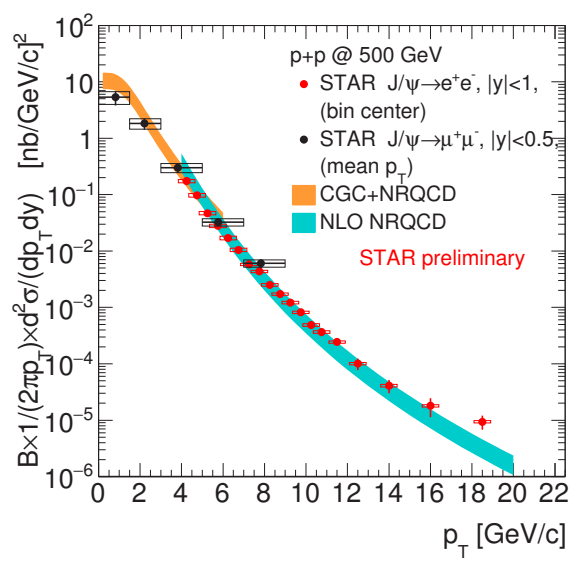

Figure 10. $J / \psi$ cross section at $\sqrt{s_{N N}}=500 \mathrm{GeV}$ as a function of $p_{T}$ in the di-muon decay channel (black circle) and in the di-electron decay channel (red circle).

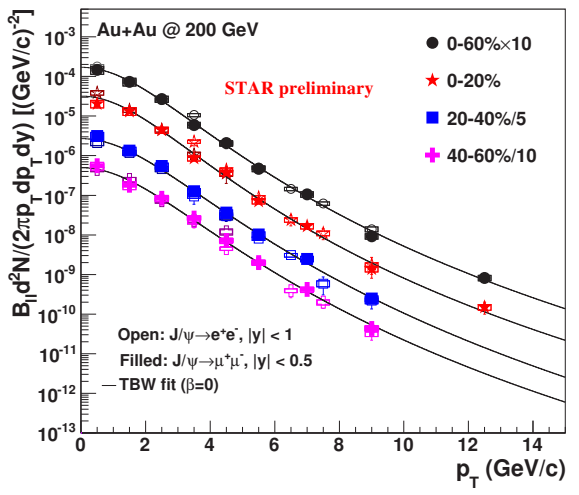

Figure 11. The $J / \psi$ invariant yields in $\mathrm{Au}+\mathrm{Au}$ collisions at $\sqrt{s_{N N}}=200 \mathrm{GeV}$ scaled by branching ratio $B_{l l}$ as a function of $p_{T}$ from di-muon decay channel (solid points) and from di-electron decay channel (open points).

Figure 10 shows the results on $J / \psi$ production cross section in $\mathrm{p}+\mathrm{p}$ collision at $\sqrt{s}=500 \mathrm{GeV}$ as a function of $p_{T}$ up to $p_{T}=20 \mathrm{GeV} / c$. The MTD measurements from the di-muon decay channel extend the $p_{T}$ reach down to $0 \mathrm{GeV} / c$. They are compatible with previous measurement from di-electron decay channel in the overlapping $p_{T}$ region. The experimental data are in a good agreement with CGC+NRQCD calculations at low $p_{T}$ [28] and with NLO NRQCD calculations at high $p_{T}$ [29].

STAR experiment has also measured $J / \psi$ invariant yields in $\mathrm{Au}+\mathrm{Au}$ collisions at $\sqrt{s_{N N}}=200 \mathrm{GeV}$ previously in di-electron and recently in di-muon channel. In Figure 11 are shown invariant yields of $J / \psi$ in di-muon channel as a function of $p_{T}$ together with results from di-electron decay channel for different collision centralities. These new results from the di-muon decay channel are consistent with the STAR published results from the di-electron decay channel $[27,30]$ within uncertainties. 


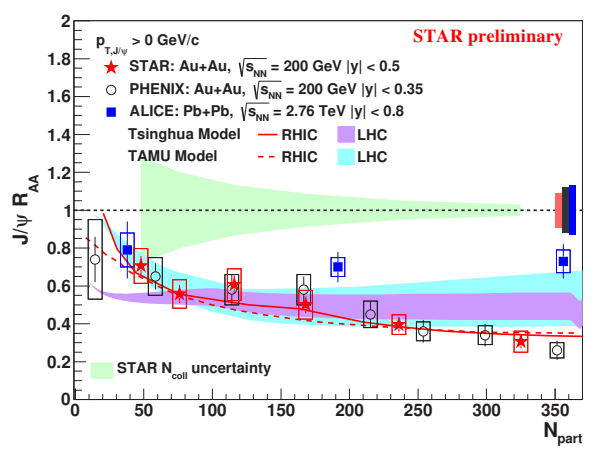

Figure 12. $J / \psi$ nuclear modification factor for integrated $p_{T}$ in $\sqrt{s_{N N}}=200 \mathrm{GeV} \mathrm{Au}+\mathrm{Au}$ collisions compared to LHC results as function of $N_{\text {part }}$.

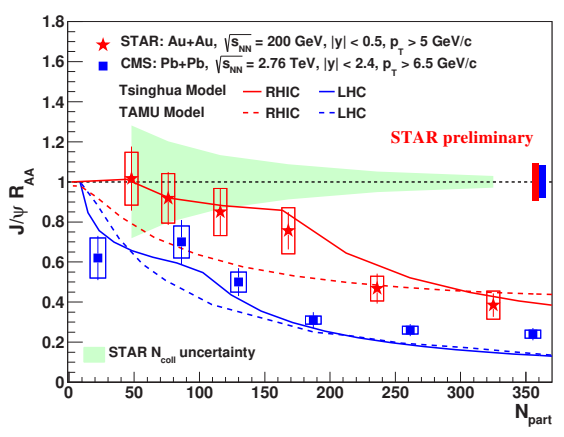

Figure 13. The $J / \psi$ nuclear modification factor for integrated $p_{T}>5 \mathrm{GeV} / \mathrm{c}$ in $\sqrt{s_{N N}}=200 \mathrm{GeV}$ $\mathrm{Au}+\mathrm{Au}$ collisions compared to LHC results as function of $N_{\text {part }}$.

In Figure 12 is shown centrality dependence of $J / \psi \mathrm{R}_{A A}=\frac{\sigma_{\text {inel }} d^{2} N_{A A} / d y d p_{T}}{\left\langle N_{\text {coll }}\right\rangle d^{2} \sigma_{p p} / d y d p_{T}}$ from the MTD data (red stars) for $p_{T}>0 \mathrm{GeV} / c$ and in Figure 13 for $p_{T}>5 \mathrm{GeV} / c$. The STAR results are compared to PHENIX results at the same collision energy (black open circles) [31], as well as results from ALICE [32] and CMS [33] in $\mathrm{Pb}+\mathrm{Pb}$ collisions at $\sqrt{s_{N N}}=2.76 \mathrm{TeV}$ (blue solid circles). For the integrated $p_{T}$ the STAR results are consistent with the PHENIX results (black open circles) showing a decrease with increasing centrality. This differs from ALICE results which are almost centrality independent and less suppressed. Such difference can be possibly explained by larger regeneration contributions at low $p_{T}$ due to higher $c \bar{c}$ yields at LHC collision energies. On the other hand, for $p_{T}>5 \mathrm{GeV} / \mathrm{c}$ in Figure 13 RHIC data show less suppression compared to LHC for all centralities. This can be an effect of higher energy density and temperature of the system created at the LHC which in turn leads to stronger $J / \psi$ suppression.

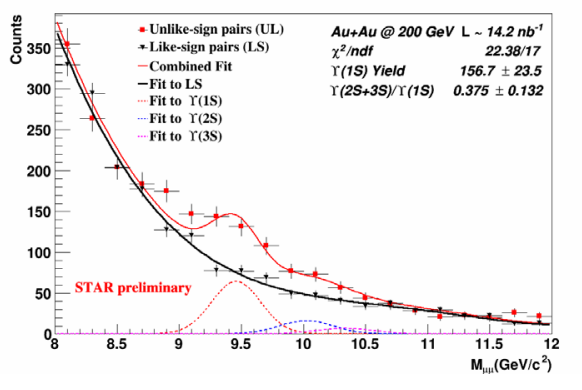

Figure 14. $\Upsilon$ signal from di-muon decay channel.

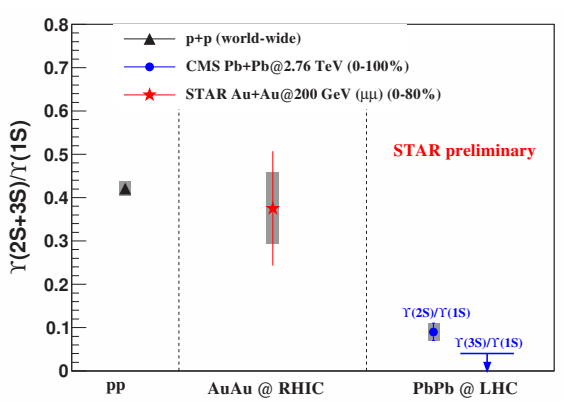

Figure 15. Comparison of $\Upsilon(2 S+3 S) / \Upsilon(1 S)$ in $\mathrm{p}+\mathrm{p}$ and heavy-ion collisions at RHIC and LHC.

Compared to $J / \psi$, measurements with $\Upsilon$ states have the advantage that they do not suffer from the regeneration contribution since $b \bar{b}$ pairs have much smaller cross section at RHIC energies when compared to $c \bar{c}$ pairs. In Figure 14 is shown reconstructed $\Upsilon$ signal from di-muon decay channel 
in $\mathrm{Au}+\mathrm{Au}$ collisions at $\sqrt{s_{N N}}=200 \mathrm{GeV}$. A signal of $\Upsilon(2 \mathrm{~S}+3 \mathrm{~S})$ in the di-muon decay channel can be observed. Figure 15 shows the $\Upsilon(2 S+3 S) / \Upsilon(1 S)$ ratio which is obtained by simultaneous fit to the like-sign and unlike-sign distributions. The obtained ratio is compared with the world-wide $p+p$ data [34] and results from CMS [35, 36]. The preliminary results indicate that a there less melting of $\Upsilon(2 S+3 S)$ relative to $\Upsilon(1 S)$ at $\mathrm{RHIC}$.

\section{Summary and outlook}

STAR experiment at RHIC has carried out the Beam Energy Scan program during which data were collected at seven different collision energies in order to systematically study the phase diagram by changing baryon chemical potential $\left(\mu_{B}\right)$ and temperature of the created matter. This extremely successful period has brought new insights and understanding of the QCD phase diagram. The BES provided first indications that the transition from hadronic to quark-gluon degrees of freedom occurs within the collision energy range of $10 \leq \sqrt{s_{N N}} \leq 20 \mathrm{GeV}$ which is well within a reach of the RHIC collider. This offers a unique opportunity to probe QCD matter properties from below to above the transition.

Moreover, STAR has successfully completed two major detector upgrades which greatly boosted its heavy flavor program. High resolution Heavy Flavor Tracker allowed to perform first analyses with topologically reconstructed open-charm mesons showing that there is a significant flow of charmed quarks in the medium. The presented results of quarkonium suppression from Muon Telescope Detector in di-muon decay channel indicate melting of $J / \psi$ and $\Upsilon$ states at RHIC top energy.

It is clear that more systematic studies are needed in the region of collision energies of interest where the current results indicate the onset of the phase transition and possibly location of the critical point. With this motivation RHIC will be in 2019-2020 conducting a second phase of the Beam Energy Scan (BES-II). This program will take advantage of RHIC's increased luminosity thanks to electron cooling and STAR is hence expected to collect high statistics data for 5-20 GeV Au+Au collisions. Meanwhile, STAR will also carry out an inner-TPC (iTPC) upgrade, which will extend the pseudo-rapidity coverage, increase low- $p_{T}$ reach in tracking of particles for QGP bulk property study, and improve the $\mathrm{dE} / \mathrm{dx}$ resolution of proton/kaon separations at high $p_{T}$ for jet fragmentation measurements. The refined high-precision measurements will enable BES-II to further improve our understanding of the nuclear matter phase diagram.

\section{Acknowledgement}

This work was supported by the grant No. 13-20841S of Grant Agency of the Czech Republic and project No. LM2015054 of Czech Ministry of Education, Youth and Sports.

\section{References}

[1] J. Adams et al. (STAR), Nucl. Phys. A757, 102 (2005), nucl-ex/0501009

[2] K. Adcox et al. (PHENIX), Nucl. Phys. A757, 184 (2005), nucl-ex/0410003

[3] L. Ruan et al., J. Phys. G36, 095001 (2009), 0904 . 3774

[4] G. Contin (STAR), Nucl. Instrum. Meth. A831, 7 (2016)

[5] P. Braun-Munzinger, J. Wambach, Rev. Mod. Phys. 81, 1031 (2009), 0801.4256

[6] B. Mohanty, New J. Phys. 13, 065031 (2011), 1102 . 2495

[7] S. Horvat (STAR), Nucl. Phys. A956, 838 (2016), 1601.01644 
[8] K. Aamodt et al. (ALICE), Phys. Rev. Lett. 107, 032301 (2011), 1105. 3865

[9] J. Auvinen, H. Petersen, Phys. Rev. C88, 064908 (2013), 1310. 1764

[10] L. Adamczyk et al. (STAR), Phys. Rev. Lett. 116, 112302 (2016), 1601.01999

[11] H. Sorge, Phys. Rev. Lett. 82, 2048 (1999), nucl-th/9812057

[12] L. Adamczyk et al. (STAR), Phys. Rev. Lett. 112, 162301 (2014), 1401.3043

[13] Y. Nara, H. Niemi, A. Ohnishi, H. Stoecker, Phys. Rev. C94, 034906 (2016), 1601.07692

[14] S. Singha (STAR) (2016), 1610.07423

[15] Y. Aoki, G. Endrodi, Z. Fodor, S.D. Katz, K.K. Szabo, Nature 443, 675 (2006), hep-lat/0611014

[16] M.A. Stephanov, Phys. Rev. Lett. 102, 032301 (2009), 0809.3450

[17] M. Asakawa, S. Ejiri, M. Kitazawa, Phys. Rev. Lett. 103, 262301 (2009), 0904.2089

[18] M.A. Stephanov, Phys. Rev. Lett. 107, 052301 (2011), 1104.1627

[19] M.R. Lomnitz (STAR), Nucl. Phys. A956, 256 (2016), 1601.00743

[20] M.R. Lomnitz, these proceedings

[21] L. Adamczyk et al. (STAR), Phys. Rev. Lett. 113, 142301 (2014), 1404.6185

[22] L. He (STAR), J. Phys. Conf. Ser. 736, 012007 (2016)

[23] S. Cao, G.Y. Qin, S.A. Bass, Phys. Rev. C88, 044907 (2013), 1308.0617

[24] M. He, R.J. Fries, R. Rapp, Phys. Rev. C86, 014903 (2012), 1106.6006

[25] M. Nahrgang, J. Aichelin, S. Bass, P.B. Gossiaux, K. Werner, Phys. Rev. C91, 014904 (2015), 1410.5396

[26] T. Matsui, H. Satz, Phys.Lett. B178, 416 (1986)

[27] L. Adamczyk et al. (STAR), Phys.Lett. B722, 55 (2013), 1208. 2736

[28] Y.Q. Ma, R. Venugopalan, Phys. Rev. Lett. 113, 192301 (2014), 1408.4075

[29] H.S. Shao, H. Han, Y.Q. Ma, C. Meng, Y.J. Zhang, K.T. Chao, JHEP 05, 103 (2015), 1411.3300

[30] L. Adamczyk et al. (STAR), Phys.Rev. C90, 024906 (2014), 1310. 3563

[31] A. Adare et al. (PHENIX), Phys. Rev. Lett. 98, 232301 (2007), nucl-ex/0611020

[32] B.B. Abelev et al. (ALICE), Phys. Lett. B734, 314 (2014), 1311.0214

[33] S. Chatrchyan et al. (CMS), JHEP 05, 063 (2012), 1201.5069

[34] W. Zha, C. Yang, B. Huang, L. Ruan, S. Yang, Z. Tang, Z. Xu, Phys. Rev. C88, 067901 (2013), 1308.4720

[35] S. Chatrchyan et al. (CMS), Phys. Rev. Lett. 109, 222301 (2012), 1208.2826

[36] S. Chatrchyan et al. (CMS), JHEP 04, 103 (2014), 1312.6300 